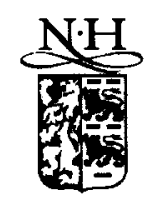

ELSEVIER

\title{
A two-dimensional model for quasigeostrophic flow: comparison with the two-dimensional Euler flow
}

\author{
Andrew J. Majda, Esteban G. Tabak * \\ Courant Institute of Mathematical Sciences, New York University, 25I Mercer Street, New York, NY 10012, USA.
}

\begin{abstract}
A simple two-dimensional model for quasigeostrophic flow is contrasted with the two-dimensional incompressible Euler equations. The model arises under the assumptions of fast rotation, uniform stratification and uniform potential vorticity. It is found that the more local feed-back of the quasigeostrophic model gives rise to strongly nonlinear front formation, as opposed to two-dimensional Euler, where the steepening process of mature fronts obeys a nonlocal, nearly linear mechanism.
\end{abstract}

Keywords: Geostrophic balance; Frontogenesis; Euler equations; Vortex stretching; Singular behavior

\section{Introduction}

The behavior of the ocean and the atmosphere in the mid-latitudes may be considered as a small departure from the fast rotation of the Earth as a solid body. This provides the ground for the quasigeostrophic approximation, which formally expands a solution to the full equations of fluid dynamics around such uniformly rotating state (see for instance [11]). The resulting equations, though much simpler than the full set, are still complex enough that it is not always clear what they imply about the nature of their solutions. Therefore further simplifications have been sought in particular contexts, looking for more tractable models.

We study one such model, based on postulating a uniformly stratified ocean or atmosphere, and a nearly uniform potential vorticity. This model has been proposed for atmospheric turbulence by Blu-

\footnotetext{
* Corresponding author. Tel. (212) 998-3088; fax: (212) 9954121; e-mail: tabak@cims.nyu.edu.
}

men in [2], a study continued by Pierrehumbert et al. [12]. Constantin, et al.[5,6] have applied it to study frontogenesis, where they explored the model's strong mathematical connections to the three-dimensional (3D) incompressible Euler equations.

In this article, we compare the (2D) quasigeostrophic (QG) model with the 2D incompressible Euler equations, to which it is linked by a formal resemblance. Interesting elements of such comparison have been studied by Held, et al. [8]. Here, we concentrate on the similarities and differences in the process of front formation. It has been shown in [6] that front formation in the $2 \mathrm{D} Q \mathrm{QG}$ model is analogous in a deep sense to vortex stretching in 3D Euler. However, the model is still 2D and, as such, shares many features of $2 \mathrm{D}$ Euler, particularly in what regards conserved quantities.

The article is organized as follows: In Section 2, a sketch of the derivation of the 2D QG model is provided. In Section 3, the model is compared to 2D Euler, with emphasis on the conserved quantities and the 
scaling properties of the stretching term. In Section 3, a numerical example shows how the different scaling properties of both models affect the mechanisms ruling the evolution of mature fronts, which are mostly linear in 2D Euler and strongly nonlinear in the QG model. The conclusions of this comparison are summarized in Section 4.

\section{A two-dimensional model for quasigeostrophic flow}

The long-scale dynamics of the atmosphere and the ocean in the mid-latitudes is governed by a balance between the Coriolis force and the pressure gradients. The mathematical expression of this "geostrophic" balance is

$-f v=P_{x}, \quad f u=P_{y}$,

where $u$ and $v$ are the $x$ - and $y$-components of the velocity, $P$ is the pressure and $f$ the vertical component of the rotation of the Earth. It follows from (1) that the horizontal component of the velocity field is tangent to the isobars. Given any pressure distribution, the equations in (1) allow us to compute the velocity field. However, these equations do not provide any information on the dynamics of the pressure distribution itself. In order to find equations ruling the evolution of the pressure, one needs to go one order further in a formal expansion in the small parameter $\epsilon$, the Rossby number, which measures the ratio of the inertial terms to the Coriolis force. Such an expansion yields (see for instance [11]), at zeroth order, the geostrophic balance, and at first order, the conservation of potential vorticity

$\frac{\mathrm{D} q}{\mathrm{D} t}=0$,

where $\mathrm{D} / \mathrm{D} t$ stands for the horizontal total derivative $\partial / \partial t+u \partial / \partial x+v \partial / \partial y$ and $q$ for the potential vorticity

$q=\frac{\partial^{2} \psi}{\partial x^{2}}+\frac{\partial^{2} \psi}{\partial y^{2}}+\frac{\partial}{\partial z}\left(\left(\frac{f}{N}\right)^{2} \frac{\partial \psi}{\partial z}\right)$.

Here $\psi$ is the horizontal stream function and $N$ the buoyancy frequency.
The simplified model with which we will concern ourselves here considers solutions in which the potential vorticity $q$ is initially constant and, due to (2), remains constant forever. For simplicity, we will make this constant equal to zero, and we will assume that the value of $N$ in (3) is uniform and constant, which, after rescaling $z$ in (3), yields Laplace's equation for $\psi$ :

$\frac{\partial^{2} \psi}{\partial x^{2}}+\frac{\partial^{2} \psi}{\partial y^{2}}+\frac{\partial^{2} \psi}{\partial z^{2}}=0$.

Next we focus on the surface of the Earth (or the bottom of the sea), assumed flat, where the velocity field is purely horizontal. Invoking (1), we can identify the pressure with a stream function, defined so that

$u=\nabla^{\perp} \psi$,

where the perpendicular gradient $\nabla^{\perp}$ is the vector with components $\left(-\partial_{y}, \partial_{x}\right)$. But the vertical derivative of the pressure is proportional to the potential temperature $\theta$, so

$\frac{\partial \psi}{\partial z}=\theta$,

and the potential temperature itself is advected by the fluid:

$\frac{\mathrm{D} \theta}{\mathrm{D} t}=0$.

Eqs. (5) and (7) involve the horizontal derivatives of $\psi$, while Eq. (6) involves its vertical derivative. These derivatives, however, are related by a Neumann map, since $\psi$ satisfies Laplace's equation (4) for $z>0$. Then

$\theta=\frac{\partial \psi}{\partial z}=(-\Delta)^{1 / 2} \psi$,

where $\Delta$ stands for the 2D horizontal Laplacian, and its square root is the pseudo-differential operator defined in Fourier space by $(-\Delta)^{\widehat{1 / 2}} \psi(k)=|k| \widehat{\psi}(k)$. Putting (7) and (8) together, we get the following system for $\theta$ and $\psi$ :

$\frac{\mathrm{D} \theta}{\mathrm{D} t}=\frac{\partial \theta}{\partial t}+v \cdot \nabla \theta=0, \quad v=\nabla^{\perp} \psi=\left(-\psi_{y}, \psi_{x}\right)$, $(-\Delta)^{1 / 2} \psi=\theta$. 
This is the 2D QG model, previously studied in $[2,6,8]$, with which we will concern ourselves in this article.

\section{Comparison with two-dimensional Euler}

The equations in (9) are formally analogous to the 2D incompressible Euler equations in vorticity form. In this section, we investigate to which extent this analogy holds.

The $2 \mathrm{D}$ incompressible Euler equations may be written in the form

$\frac{\mathrm{D} \omega}{\mathrm{D} t}=\frac{\partial \omega}{\partial t}+\boldsymbol{v} \cdot \nabla \omega=0, \quad \boldsymbol{v}=\nabla^{\perp} \psi, \quad \Delta \psi=\omega$.

Here $\omega$ is the vertical component of the vorticity vector

$w=v_{x}-u_{y}=\psi_{x x}+\psi_{y y}$.

The formal analogy between (9) and (10) is clear. In both cases, a scalar is advected by a divergence-free velocity field:

$\frac{\mathrm{D} \theta}{\mathrm{D} t}=0, \quad \frac{\mathrm{D} \omega}{\mathrm{D} t}=0$.

$v=\nabla^{\perp} \psi$.

This already implies that, for any function $F$, the following integrals over the whole domain are conserved:

$\int F(\theta), \quad \int F(\omega)$.

In particular, choosing $F$ as the characteristic function of an interval, we see that both equations preserve the area between two contour lines of the advected scalar.

In both cases the scalar is "active" [3], in the sense that its distribution determines the velocity field. There exists an important difference between the two cases, however, in the relation between the active scalar and the stream function:

$(-\Delta)^{1 / 2} \psi=\theta, \quad \Delta \psi=\omega$.

These relations can be represented in Fourier space as

$|k| \hat{\psi}=\hat{\theta}, \quad|k|^{2} \hat{\psi}=\hat{\omega}$

and, in physical space, as

$$
\begin{aligned}
& \psi=-\int \frac{\theta(x+y)}{|y|} \mathrm{d} y, \\
& \psi=\frac{1}{2 \pi} \int \omega(x+y) \log |y| \mathrm{d} y .
\end{aligned}
$$

For the QG model, the Fourier modes of the velocity and the potential temperature satisfy

$|\widehat{v}|^{2}=|\widehat{\theta}|^{2}$.

Therefore the kinetic energy

$\int \frac{1}{2}|v|^{2}$

is conserved, as follows from applying Plancherel's formula to write the energy in Fourier space, invoking (18) to translate the $\hat{v}$ 's into $\hat{\theta}$ 's, returning to physical space, and utilizing (14) with the particular choice $F=\frac{1}{2} \theta^{2}$. Thus, the energy for the QG model is actually a redundant conserved quantity deduced from (14). On the other hand, the kinetic energy is also preserved by $2 \mathrm{D}$ Euler, but for a different reason. For $2 \mathrm{D}$ Euler, we may integrate the kinetic energy by parts into

$\int \psi \omega$

for which it is a well-known straightforward exercise to show conservation. A similar computation for the QG model shows that the quantity

$\int \psi \theta$

is conserved. This quantity, however, is not the kinetic energy; thus, the structure of the conserved quantities in both equations is similar.

The next level of analogies and differences concerns the possibility of development of singularities. For 2D Euler, as is well-known classically [10], no finite time singularities may arise from smooth data. For the QG equations, however, the possibility of singularities is an open question, related, as we will see below, to that of singularities of 3D Euler. As both $\theta$ and $\omega$ are advected by the fluid, it is not these quantities but their derivatives which might blow-up. As it turns out (see [1] for a similar constraint on 3D Euler), it is enough to look at first derivatives (see [6] for the proof.) In 
other words, if the QG model (9) develops finite time singularities, the gradient of $\theta$ has to blow-up. In face of this fact, let us write down the equations that the gradients of $\theta$ and $\omega$ satisfy. These equations, which can be found simply differentiating (9) and (10), are

$\frac{\mathrm{D} \nabla^{\perp} \theta}{\mathrm{D} t}=\nabla \boldsymbol{v} \cdot \nabla^{\perp} \theta$

and

$\frac{\mathrm{D} \nabla^{\perp} \omega}{\mathrm{D} t}=\nabla v \cdot \nabla^{\perp} \omega$.

These equations represent convection of the gradients of $\theta$ and $\omega$, and "stretching" caused by the deformation matrices $\nabla v$. These matrices have the representations, for the QG case,

$\nabla v(x)=-P \cdot V \cdot \int \nabla^{\perp} \theta(x+y) \otimes \nabla\left(\frac{1}{|y|}\right) \mathrm{d} y$

and for 2D Euler,

$\nabla v(x)=\frac{1}{2 \pi} \int \nabla^{\perp} \omega(x+y) \otimes \nabla(\log |y|) \mathrm{d} y$.

The relation between stretched and stretching variables is given, in the QG case, by a singular, principal value integral, and for 2D Euler, by a convolution with a locally integrable kernel. Thus singular behavior appears more likely in the QG model, where potential singularities provoke a stronger feed-back.

This difference in feed-backs can be observed qualitatively in the relations (16) between the active scalar and the stream function in Fourier space. The longwave (small $|k|$ ) behavior of $\omega$ affects $\psi$ more strongly than $\theta$ does; for short waves, however, as the ones corresponding to a localized singularity, these effects switch, $\theta$ acting far more strongly than $\omega$ on $\psi$.

Surprisingly, this more local feed-back of the QG model is analogous not to 2D but to 3D Euler. To see this, let us write the 3D incompressible Euler equations in stream-vorticity formulation:

$$
\frac{\mathrm{D} \omega}{\mathrm{D} t}=(\nabla v) \boldsymbol{\omega},
$$

where $\boldsymbol{\omega}$ is the vorticity vector, and $\boldsymbol{v}$, the velocity, is a 3D divergence-free vector field. The vorticity is the curl of the velocity field: $\omega=\nabla \times v$.

Conversely, $v$ can be determined from $\boldsymbol{\omega}$ by the BiotSavart law

$\boldsymbol{v}(x)=-\frac{1}{4 \pi} \int\left(\nabla^{\perp} \frac{1}{|y|}\right) \times \boldsymbol{\omega}(x+y) \mathrm{d} y$.

Then the gradient of $v$ is given by a singular principal value integral operator acting on $\omega$. This makes (23) analogous to (19), if we identify $\boldsymbol{\omega}$ with $\nabla^{\perp} \theta$. This analogy has been carried out in great detail in [6], to which we refer the interested reader.

Therefore, the formal analogy between the 2D QG model and 2D Euler breaks down when one studies the development of sharp fronts (elongated vortex filaments in 2D Euler.) The nonlinear feed-back is far less likely to saturate at an early stage in this development for the QG model than for 2D Euler. Indeed, the mathematical structure of the QG model is surprisingly close to that of 3D Euler, for which the collapse of vortex tubes is a strongly nonlinear event. This collapse may even give rise to a blow-up of vorticity in finite time, as some numerical evidence [9] seems to indicate. Similar evidence has been collected in [6] for the much simpler QG model.

\section{A numerical example}

How does the more local feed-back of the QG model manifest itself in actual solutions? To answer this question, we have run a numerical example, contrasting the QG model with 2D Euler. The initial data are the same ones that we used in [5] to study front formation, for the QG model

$\theta(x, y, 0)=\sin (x) \sin (y)+\cos (y)$

and for 2D Euler

$\omega(x, y, 0)=\sin (x) \sin (y)+\cos (y)$.

We solved Eqs. (9) and (10) with these initial data, using a spectral collocation method (see [5,6].) The velocities $v(\theta)$ and $v(\omega)$ were computed in Fourier space, using (13) and (16), and the products $v \cdot \nabla \theta$ and $v \cdot \nabla \omega$ were computed in physical space. An exponential filter of high frequencies, like the one developed in 


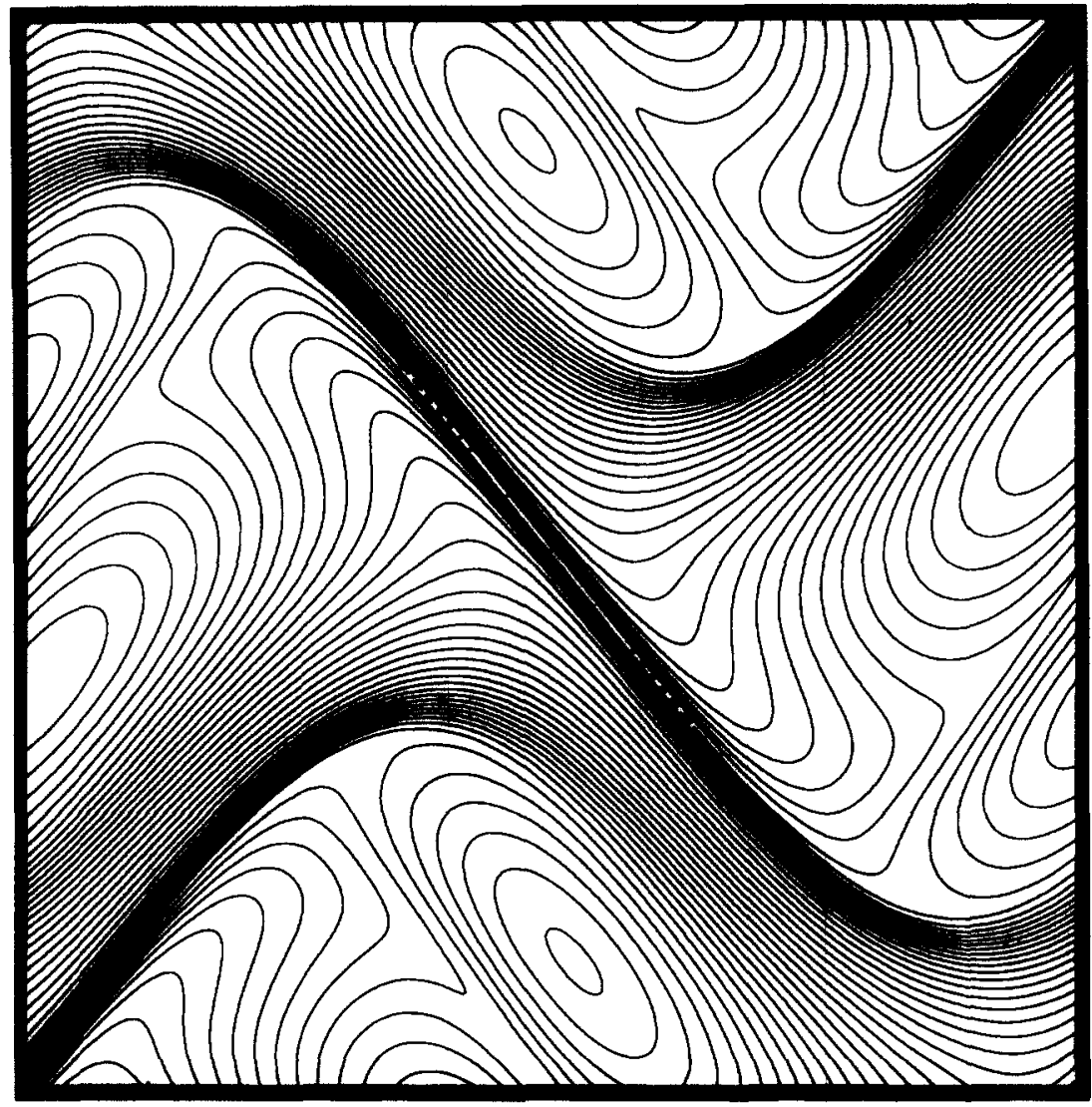

Fig. 1. Contour lines of $\theta$ for the 2D QG model, at time $t=6$, with initial data $\theta_{0}=\cos (y)+\sin (x) \sin (y)$. A grid of $512 \times 512$ points has been used for this computation.

[7], was used to avoid aliasing effects. A fourth-order Runge-Kutta method was adopted as time-advancing routine.

The contour lines of $\theta$ and $\omega$ at time $t=6$ are displayed in Figs. 1 and 2. The smooth data (26) and (27), with a saddle point at the origin, soon develop sharp elongated fronts, resembling antiparallel vortex filaments in 3D Euler. At time $t=6$, both models have developed fronts which we may call "mature", in the sense that, although both fronts continue steepening, they do so by mechanisms which remain stable over relatively large time intervals. Our claim is that these mechanisms are nonlocal and linear for 2D Euler, and local and nonlinear for the QG model. To substantiate this claim, we observe that the filaments near the origin for 2D Euler in Fig. 2 are nearly perfectly antiparallel, having essentially forgotten the two dimensionality associated with the initial saddle point. Instead, the filaments in Fig. 1, though straightening up, do so in a manner which preserves more of the initial two dimensionality.

It has been shown rigorously in [6] that, for nonlinearity to survive a stretching and straightening process, this process has to be balanced by a packing of contour lines in such a way that $2 \mathrm{D}$ effects remain active. Although it is very difficult to measure this subtle balance in fronts which are barely resolved by the computation, it is apparent that the ones in Fig. 1 are far more likely to satisfy such balance than those in Fig. 2. The intuition behind this geometrical balance is the following: A purely 1D front has an associated velocity field which runs parallel to the front, and cannot therefore affect it. Par consequence, the continuing stretching of fronts such as the one in Fig. 2 has 


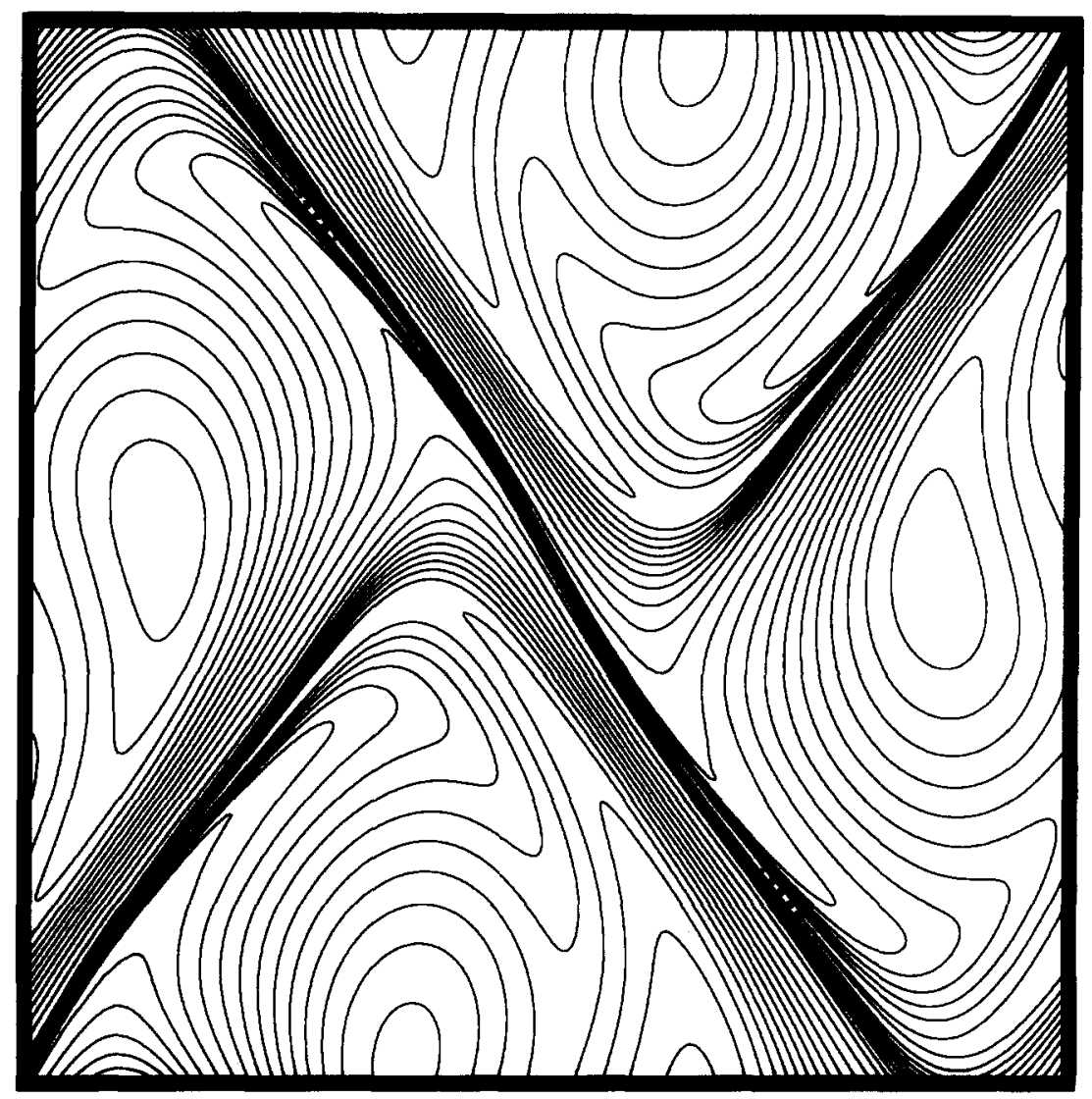

Fig. 2. Contour lines of $\omega$ for $2 \mathrm{D}$ Euler, at time $t=6$, with initial data $\omega_{0}=\cos (y)+\sin (x) \sin (y)$, computed on a grid of $512 \times 512$ points.

to be caused by nonlocal effects. Instead, if a front is nearly $1 \mathrm{D}$ but also very steep, in such a way that the resulting velocity field transversal to the front does not vanish, a local, nonlinear self-feeding process remains possible.

In order to go beyond the qualitative comparison of contour lines in Figs. 1 and 2, we use rigorous mathematical diagnostics for singularity formation (see $[6,10]$ ). We plot in Figs. $3-5$ the time evolution of the maximum gradient of $\theta$ and $\omega$. In Fig. 3, we see how both gradients grow at an increasingly fast rate. The fact that $\nabla \omega$ in $2 \mathrm{D}$ Euler grows initially much faster than $\nabla \theta$ in the QG model is due to the larger velocities induced nonlocally in the former (compare (21) and (22).) Fig. 4 displays the same evolutions in a semi-logarithmic scale. We see here how the initial nonlinear growth in 2D Euler rapidly saturates, giving rise to a purely linear, exponential growth, manifested through a straight line in the semi-logarithmic plot. This exponential growth shows that, for 2D Euler, our numerical results are consistent with the fact that no singularities occur at finite time (see [10]). The growth in the QG model, though initially slower than its 2D Euler counterpart, is always strongly nonlinear and faster than exponential. Finally, Fig. 5 shows the same evolutions in a $\log$-log scale, with the origin of time at $t=8.25$, the conjectured critical time of this experiment, in which the solution to the QG model may become singular. Now the growth in the QG model fits a straight line with slope $-\frac{5}{3}$, corresponding to a singular behavior of $\nabla^{\perp} \theta$ proportional to $(8.25-t)^{-5 / 3}$. The exponential curve for 2D Euler bends downward in this plot instead. 


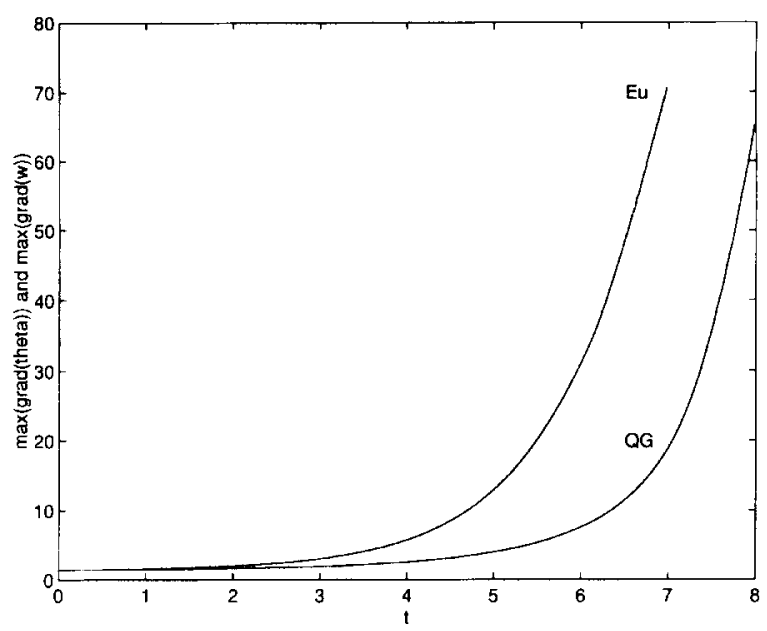

Fig. 3. Maximum values of $\left|\nabla^{\perp} \theta\right|$ and $\left|\nabla^{\perp} \omega\right|$ as functions of time. The rate of growth of $\left|\nabla^{\perp} \omega\right|$ is initially faster.

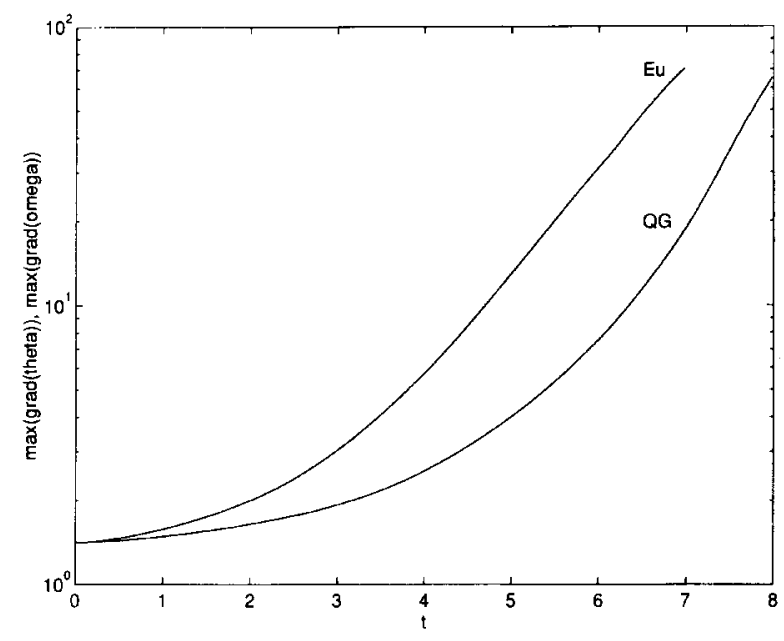

Fig. 4. Same as Fig. 3, in semi-logarithmic scale. The growth of $\left|\nabla^{\perp} \omega\right|$ soon saturates to an exponential; the one of $\left|\nabla^{\perp} \theta\right|$, instead, is always faster than exponential.

\section{Conclusions}

The incompressible 2D Euler equations and the 2D QG model, despite their formal similarity and a large number of common conserved quantities, have fronts with very different behavior. The stronger local feedback of the QG model gives rise to a sustained nonlinear steepening of the fronts; similar fronts in 2D Euler end up growing only linearly, due to velocity fields created nonlocally. Thus, in their final stages of devel-

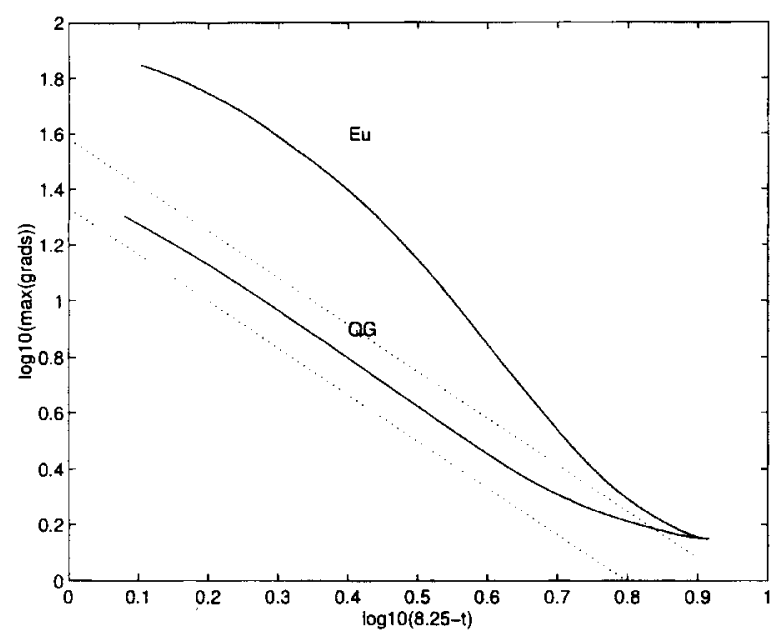

Fig. 5. Same as Figs. 3 and 4 , in $\log -\log$ scale, showing the behavior of $\left|\nabla^{\perp} \theta\right|$, which grows like $1 /(8.25-t)^{5 / 3}$. This seems to indicate a collapse near time $t=8.25$. However, the grid resolution fails before that time.

opment, the QG fronts behave in a way much more reminiscent of stretching vortex filaments in 3D Euler.

In this article, we have looked at the analytical similarities and differences between 2D Euler and the QG model, and performed a numerical experiment to determine how the differences manifest themselves in the process of front formation. Theory and experiments agree, in that the stronger nonlocal effects on the induced velocity field of 2D Euler give rise initially to faster growing fronts, which switch very early to a nearly linear regime, while the QG fronts, exhibiting an initially slower rate of growth, are capable of sustaining a long nonlinear self-stretching process, which may end up in a finite time collapse. Additional geometric, analytic and numerical diagnostics of this strongly nonlinear behavior can be found in [6].

\section{Acknowledgements}

A.J. Majda was partially supported by grants NSF DMS-99301094, ARO DAAL03-92-G-0010, ONR N00014-89-J-1044.P00003 and DARPA N00014-92J-1796; E.G. Tabak was partially supported by grant NSF DMS-9501073. 


\section{References}

[1] T. Beale, T. Kato and A. Majda, Remarks on breakdown of smooth solutions for the three-dimensional Euler equations, Commun. Math. Phys. 94 (1984) 61-66.

[2] W. Blumen, Uniform potential vorticity flow. Part I. Theory of wave interactions and two-dimensional turbulence, J. Atmos. Sci. 35 (1978) 774-783.

[3] P. Constantin, Geometric Statistics in Turbulence, SIAM Rev. 36 73-98.

[4] P. Constantin, P.D. Lax and A. Majda, A simple onedimensional model for the three-dimensional vorticity equation, Commin. Pure Appl. Math. 38 (1985) 715-724.

[5] P. Constantin, A. Majda and E.G. Tabak, Singular front formation in a model for quasigeostrophic flow, Phys. Fluids 6 (1) (1994) 9-11.

[6] P. Constantin, A. Majda and E.G. Tabak, Formation of strong fronts in the 2-D quasigeostrophic thermal active scalar, Nonlinearity 7 (1994) 1495-1533.
[7] E. Weinan and C. Shu, Effective equations and inverse cascade theory for Kolmogorov flows, Phys. Fluids A 5 (4) (1993) 998-1010.

[8] I.M. Held, R.T. Pierrehumbert, S.T. Garner and K.L. Swanson, Surface quasi-geostrophic dynamics, J. Fluid Mech. 282 (1995) 1-20.

[9] R. Kerr, Evidence for a singularity of the threedimensional, incompressible Euler equations, Phys. Fluids A 5 (7) (1993) 1725-1746.

[10] A. Majda and A. Bertozzi, Vorticity and the Mathematical Theory of Incompressible Flow, to appear.

[11] J. Pedlosky, Geophysical Fluid Dynamics, (Springer, New York, 1987) pp.345-368 and 653-670.

[12] R.T. Pierrehumbert, I.M. Held and K. Swanson, Spectra of local and nonlocal two dimensional turbulence, Chaos, Solitons, and Fractals 4 (1994) 1111-1116. 